\title{
Asociación entre el tipo de gestión escolar y el rendimiento de los estudiantes en zonas urbanas ${ }^{1}$ \\ Association between type of school and student achievement in urban areas
}

\author{
Alejandra Miranda \\ Grupo de Análisis para el Desarrollo \\ amiranda@grade.org.pe
}

Recibido: 15-5-2015

Aprobado: 28-8-2015

1. Este artículo se basa en la tesis de maestría de la autora (ver referencia). 


\title{
Resumen
}

En los últimos, años el acceso a la educación básica ha aumentado de manera significativa; sin embargo, los niveles de rendimiento siguen siendo bajos y el progreso que se ha logrado no es suficiente para garantizar una educación de calidad. En este contexto, la investigación de los factores asociados al rendimiento estudiantil se convierte en un tema relevante. Haciendo uso de una base de datos longitudinal, este trabajo se enfoca en estimar el efecto del tipo de gestión de las escuelas - públicas o privadas- ubicadas en el área urbana en el rendimiento de niños entre 7 y 8 años de edad en matemática y vocabulario (PPVT). Los resultados muestran que el asistir a una escuela privada tiene un efecto positivo y significativo en matemática pero no en vocabulario. Esto puede deberse a que el rendimiento en matemática depende en mayor medida del currículo implementado en las escuelas, mientras que el vocabulario de los niños puede estar influenciado, también, por el hogar y la familia. Finalmente, se encontró que la diferencia en el puntaje del PPVT entre los niños que asisten a instituciones educativas públicas y privadas responde, principalmente, al rendimiento previo de los niños; en cambio, en matemática, la brecha se puede explicar, sobre todo, a través de las variables asociadas al niño y la familia.

Palabras clave: gestión escolar, escuela privada, educación urbana, rendimiento del estudiante, Perú

\begin{abstract}
Over the past years, access to basic education has increased significantly. However, learning levels remain low and the progress that has been achieved is not enough to guarantee a good education. Given this context, exploring the factors associated with student achievement becomes an important issue. Using a longitudinal data base, this study seeks to estimate the effect of the type of school - public or private - in the urban area, on children's achievemnt aged 7-8 years in math and vocabulary (PPVT). Results show that attending a private school has a positive and significant effect in math but not in vocabulary. This may be because learning math depends more on the curriculum implemented in schools while children's vocabulary may also be influenced by the family. Finally, the difference in the PPVT score between children attending public and private schools is mainly explained by children's previous achievements; while in mathematics, the gap is mainly explained by variables associated to the child and his family.
\end{abstract}

Keywords: type of school, private school, urban education, student achievement, Peru 


\section{Asociación entre el tipo de gestión escolar y el rendimiento de los estudiantes en zonas urbanas}

\section{Introducción}

En los últimos años, el acceso a la educación ha aumentado de manera significativa, como lo muestra la tasa neta de asistencia y la tasa de cobertura total, las cuales, en 2013 , alcanzaron para primaria el $93,2 \%$ y $98,8 \%$ respectivamente. No obstante, la calidad de la educación, en términos del rendimiento de los estudiantes, aún no es la esperada, puesto que, si bien ha habido un aumento en el aprendizaje, los resultados en las evaluaciones nacionales e internacionales sugerirían que el rendimiento de los estudiantes aún debe mejorar.

Los resultados de la Evaluación Censal de Estudiantes $(\mathrm{ECE})^{2}$ señalan que ha habido un progreso en ambas áreas evaluadas; sin embargo, los últimos resultados (2014) muestran que el 57\% de los estudiantes se encuentra por debajo del nivel 2 (nivel satisfactorio) en Comprensión Lectora; y 74\%, en Matemática (Oficina de Medición de la Calidad de los Aprendizajes, 2015). Con respecto a las evaluaciones internacionales, el Perú ha participado en seis: tres realizadas por el Laboratorio Latinoamericano de Evaluación de Calidad (LLECE) y tres llevadas a cabo por el Programa Internacional para la Evaluación de Estudiantes (PISA). En el Tercer Estudio Regional Comparativo y Explicativo (Terce), en promedio, la mitad de los evaluados de tercer grado se ubicó en el Nivel I y en $<$ Nivel $I$, los dos niveles más bajos dentro de los cinco establecidos por el Terce $^{3}$ (Unesco, 2014). Asimismo, los resultados de PISA 2012 muestran que, en matemática, casi la mitad de los estudiantes de quinto de secundaria (47\%) se encuentra en el último nivel; y ninguno, en el nivel más alto (OECD, 2013).

A partir de estas cifras, se puede inferir que, a pesar del importante progreso en el acceso a la educación de más niños y niñas, el rendimiento sigue siendo bajo y el progreso que se ha logrado no es suficiente para garantizar una educación de calidad (Learning Metrics Task Force, 2013). En este contexto, la investigación de los factores asociados al rendimiento estudiantil se convierte en un tema relevante. Así, el estudio sobre el efecto del tipo de gestión de las escuelas - públicas o privadas-continúa siendo un tema en debate, puesto que, en las últimas décadas, el crecimiento de la oferta y la demanda de la educación privada se ha visto en aumento, particularmente, en la zona urbana, en la que se concentra el 98\% de la matrícula privada en educación básica regular (Unidad de Estadística Educativa - Escale, 2014). Este crecimiento ha ido acompañado por la idea de que la educación privada es mejor que la pública, tanto en zonas

2. Se trata de una evaluación que es llevada a cabo por el Ministerio de Educación (Minedu) de manera anual desde el año 2007 en las áreas de Matemática y Comprensión Lectora a niños de segundo grado de primaria.

3. El $45 \%, 60 \%$ y $54 \%$ de los alumnos de tercer grado se ubicaron en los 2 niveles más bajos en Lectura, Matemática y Ciencias Naturales, respectivamente. 
de nivel socioeconómico medio o alto, como en zonas marginales y emergentes para el contexto peruano (Montero y Cuenca, 2008) e internacional (Tooley y Dixon, 2003; Tooley, Dixon y Gomathi, 2007).

Dicho lo anterior, en esta investigación, se plantean dos objetivos. El primero es identificar si existen, y en qué magnitud, diferencias significativas entre el rendimiento de estudiantes de 7 y 8 años de edad que asisten a escuelas públicas y el de aquellos que acuden a escuelas privadas en el área urbana, debido a que es ahí donde se concentra el $98 \%$ de colegios privados. El segundo objetivo es explicar a qué se deben estas diferencias o brechas en el rendimiento, para lo cual se calculará qué proporción se puede atribuir a características del niño y de la familia o a características la escuela. Al tratar de responder estos objetivos, la investigación contribuirá de varias maneras a la literatura existente. En primer lugar, escasos estudios en el Perú se han focalizado en descomponer la brecha de rendimiento entre niños que asisten a escuelas públicas y privadas observando qué variables son las que explican en mayor proporción las diferencias. En segundo lugar, una de las limitaciones que presentan muchos estudios es el uso de bases de corte transversal. Sin embargo, al usar una base de datos longitudinal -como la del estudio Niños del Milenio ${ }^{4}$-, se podrá tener información de los insumos de las familias a lo largo del tiempo, lo cual permite obtener estimadores más confiables.

\section{Educación pública, privada y rendimiento}

A partir de los años noventa, el Perú ha venido experimentando un continuo crecimiento en la oferta y demanda de la educación privada: mientras en el año 1993 la matrícula en primaria en colegios privados era 12,3\% a nivel nacional, en el año 2014, fue 25,0\% (Unidad de Estadística Educativa - Escale, s.f.). En otras palabras, en los últimos veinte años, el total de matriculados en primaria en colegios privados se duplicó. Una tendencia opuesta se ha visto en zonas rurales, donde la concentración de estudiantes se da en escuelas públicas: los matriculados en escuelas privadas eran $2,5 \%$ en el año 2003 , mientras que, en el 2014 , fue $1 \%{ }^{5}$. Estas cifras ponen en evidencia el continuo aumento de la demanda por la educación privada, la cual ha ido de la mano con la creencia de la población de que la educación privada es mejor que la pública (Montero y Cuenca, 2008). Ello se sostiene en que las escuelas privadas estarían asociadas a una mejor provisión de servicios, docentes más preparados y mejores ambiente para los estudiantes.

Guerrero et ál. (2012) encuentran, usando la Encuesta Escolar del estudio Niños del Milenio, que las escuelas privadas tienen mayor acceso a servicios

4. Para mayor información sobre el estudio Niños del Milenio, se puede consultar Young Lives (http://www.younglives.org.uk/).

156 5. Estos porcentajes se han calculado según el Censo Escolar de los años 2003 y 2014 (Unidad de Estadística Educativa - Escale, s.f.). 
básicos -como agua, desagüe, teléfono, entre otros- en comparación con las escuelas públicas urbanas. Estos factores, mediante mejores condiciones y confort, influirían en el rendimiento de los estudiantes (Rydeen, 2009; Schneider, 2002). Asimismo, usando los mismos datos, Cueto, León, Miranda y Vásquez (s.f. $a$ ) hallaron que los docentes de escuelas privadas faltan menos a sus clases, reciben más soporte pedagógico y tienen un mejor conocimiento pedagógico del contenido que los profesores de escuelas públicas urbanas. Estos aspectos son relevantes, pues el docente es un factor clave en el rendimiento de los estudiantes. Uno de los puntos centrales, en este contexto de crecimiento de la oferta de educación privada, es que este se ha dado de manera poco regulada y son pocos los estudios actuales que han analizado la educación privada y los resultados obtenidos.

\section{Revisión de literatura}

Con respecto a la literatura, hay resultados mixtos. Coleman, Hoffer y Kilgore (1982) utilizan datos de un estudio longitudinal del Centro Nacional de Estadísticas Educacionales, llevado a cabo por la Universidad de Chicago. En este estudio, recogen información de 58 mil estudiantes de secundaria en 1015 escuelas -entre públicas, privadas y católicas- para calcular las diferencias en el rendimiento de los niños en matemática, comprensión y vocabulario. Los autores encuentran que, en matemática y vocabulario, los alumnos que asisten a escuelas católicas y privadas tienen un puntaje más alto que los que asisten a escuelas públicas. Jiménez, Lockheed y Paqueo (1991), basándose en estudios de casos, comparan el rendimiento en lenguaje y matemática de los estudiantes de secundaria que asisten a escuelas públicas y privadas en Filipinas, Tanzania, Tailandia, entre otros. A partir de ello, encuentran que los estudiantes que asisten a escuelas privadas tienen un mejor rendimiento en matemática y lenguaje que los que asisten a escuelas públicas, aún luego de controlar por el ingreso de la familia, educación de la madre, ocupación del padre, etc.

En Latinoamérica, uno de los primeros estudios llevados a cabo fue el de Psacharopolus (1986), en el cual el autor analiza las diferencias en matemática y razonamiento verbal entre estudiantes de secundaria de escuelas públicas y privadas en Colombia y Tanzania. Los resultados muestran que obtienen mejores resultados los estudiantes de escuelas privadas en ambos países luego de que se controla por variables de la familia. Por su parte, Mizala, Romaguera y Reinaga (1999) examinan los factores asociados con el desempeño de alumnos bolivianos comparando el rendimiento escolar entre colegios públicos y privados. Los autores utilizan la base del Sistema de Medición y Evaluación de la Calidad de la Educación (Simecal) para los alumnos de sexto de primaria. Mediante este procedimiento, hallaron que, si bien las variables relacionadas al hogar son importantes, las características de la escuela y de los profesores también constituyen una parte relevante en el aprendizaje de los niños. Asimismo, luego de controlar por características socioeconómicas, encontraron que los 
colegios públicos presentan resultados más bajos que los de escuelas privadas. Para el caso de Chile, algunos autores han abordado este tema haciendo uso de las evaluaciones censales Simce; sin embargo, no resaltan diferencias significativas entre ambos tipos de escuela para primaria (Mizala y Romaguera, 2000) y secundaria (McEwan, 2001).

En el Perú, son varios los estudios que exploran los determinantes del rendimiento de los alumnos, entre los cuales añaden un control por el tipo de escuela. Wu (1999) utilizó la primera evaluación nacional en el Perú -sobre matemática y lenguaje-, la cual fue aplicada en 1996 a aproximadamente 50 mil estudiantes de cuarto grado de primaria. Usando un modelo jerárquico de tres niveles, el autor evidenció que los estudiantes de escuelas privadas tienen puntajes más altos que los de escuelas públicas, aun luego de controlar por características del niño, de la escuela y del departamento. En 2000, la Oficina de Medición de la Calidad de los Aprendizajes (UMC) y el Grupo de Análisis para el Desarrollo presentaron los resultados de la segunda evaluación realizada en el Perú, Crecer 1998, en la cual se evaluó a estudiantes de escuelas urbanas polidocentes completas en cuarto y sexto de primaria, así como cuarto y quinto de secundaria en matemática y lenguaje. Los autores encontraron que las diferencias del puntaje entre los alumnos de escuelas públicas y privadas eran significativas para ambas áreas evaluadas y para los cuatro grados.

La UMC (2004) utiliza los datos de la Evaluación Nacional de 2001 para cuarto de primaria y secundaria en Matemática y Comunicación Integral. Estimando un modelo multinivel, han observado que el efecto de asistir a una escuela pública es negativo y significativo en las dos áreas evaluadas para cuarto de secundaria; sin embargo, para cuarto de primaria, el efecto no es significativo. En 2005, la UMC presentó los resultados de la Evaluación Nacional 2004, que fue la cuarta evaluación que se realizó en el país. Los grados evaluados fueron segundo y sexto grado de primaria, así como tercero y quinto grado de secundaria en Matemática, Comunicación y, solo para sexto y quinto, en Formación Ciudadana. Los resultados mostraron que el porcentaje de estudiantes que alcanzaba el nivel suficiente era mayor en las escuelas privadas, en los cuatro grados, para Matemática y Comunicación.

Chávez (2001), por su parte, utiliza la base de datos de la prueba Crecer -administrada en 1998-, aplicada a estudiantes de cuarto año de primaria en centros educativos urbanos para estimar el efecto de la escuela privada en el rendimiento de matemática. Sirviéndose de un modelo de mínimos cuadrados ordinarios, así como de un modelo lineal jerárquico, encuentra que las escuelas privadas son más efectivas que las públicas. Cueto (2011) realiza un análisis sobre la base de PISA 2009, en el cual observa el rendimiento en comprensión lectora con un enfoque de equidad y oportunidades de aprendizaje. El autor encuentra que existen brechas de rendimiento notables entre los estudiantes peruanos; esto se estima mediante un modelo lineal jerárquico. Asimismo, al añadir la variable del tipo de gestión de la escuela, observa diferencias positivas 158 I y significativas, luego de controlar por características del hogar y del niño. En 
otro estudio, Cuenca (2013) analiza la situación de las escuelas públicas y privadas en Lima Metropolitana; en este, observa que el porcentaje de niños que alcanza el nivel satisfactorio en la Evaluación Censal de Estudiantes es mayor en las escuelas privadas, tanto para Comprensión Lectora como Matemática de 2007 a 2012. El autor sostiene que estas diferencias están asociadas estrechamente al contexto socioeconómico de la escuela y los alumnos, argumentando que la oferta privada es heterogénea.

Enfocándose en otros aspectos, Valdivia (2003) estima el efecto de la escuela privada polidocente completa sobre el rendimiento estudiantil, para lo cual divide la muestra en Lima y Callao, por un lado; y el resto de centro poblados, por otro. Haciendo uso de la Evaluación Nacional de Rendimiento Estudiantil en 2001, aplicada a una muestra de cuarto grado de primaria, la autora resalta que las escuelas privadas obtienen mejores resultados que las públicas sin importar el tamaño del centro poblacional en el que operan tanto en Matemática como Lenguaje; sin embargo, las escuelas públicas son mejores que las privadas en la atención de estudiantes con atraso escolar o que usan lenguas nativas en el hogar.

Finalmente, León et ál. (2015) realizan un estudio a partir de las Evaluaciones Censales de Estudiantes (ECE) de 2007 a 2012, llevadas a cabo por el Ministerio de Educación, con el fin de analizar el sistema educativo privado. Los autores dividen las escuelas privadas en cuatro tipos: privadas élite, de gestión privada, privadas tipo cadena y el resto de escuelas privadas. En este análisis, observan que, en Comprensión Lectora, las escuelas públicas tienen un menor puntaje que los cuatro tipos de escuelas privadas; no obstante, en Matemática, son las escuelas privadas las que tienen menor puntaje que las públicas, lo cual demuestra la alta heterogeneidad del sistema educativo privado, cuya oferta ha ido en aumento desde los últimos años. Asimismo, los autores encuentran que asistir a una escuela privada tiene un efecto positivo en Compresión Lectora y Matemática. A pesar de ello, al controlar por efectos fijos de la familia, los efectos desaparecen.

En resumen, la mayoría de investigaciones se enfocan en los factores asociados al rendimiento, entre los cuales incluyen el tipo de gestión escolar como una variable explicativa. Sin embargo, son pocos los que usan modelos o técnicas para corregir los posibles sesgos de asistir a una escuela pública o privada, sesgos que existen debido a que la inversión de los padres en la educación de sus hijos depende de si pueden pagarlo o no, de su motivación (algunos padres le dan más importancia a la educación que otros) y de la habilidad del propio niño (Glewee, 2002). Este estudio aborda este problema mediante el uso de un modelo de valor agregado.

\section{Objetivos}

Luego de revisar distintos estudios empíricos y observar los resultados de las pruebas de rendimiento de los últimos años, el primer objetivo de la 
investigación es estimar la asociación entre el tipo de gestión escolar -pública o privada- y el rendimiento de los estudiantes luego de controlar por características individuales y familiares. El segundo objetivo es analizar qué factores estarían explicando la brecha de rendimiento, y calcular qué porcentaje se explica por su rendimiento previo, características del niño y la familia, de la escuela.

\section{Metodología}

\subsection{Base de datos utilizada}

Los datos que se utilizan pertenecen al estudio Niños del Milenio y al Censo Escolar 2009. Niños del Milenio es un estudio longitudinal que tiene como objetivo estudiar la pobreza infantil mediante el seguimiento de 12.000 niños en 2 cohortes: la cohorte menor (nacidos en el año 2000/2001) y la cohorte mayor (nacidos en el año 1994/1995). El estudio se realiza en cuatro países -Vietnam, Etiopía, India (Estado de Andra Pradesh) y Perú- durante quince años. Hasta la fecha, se encuentran disponibles 3 rondas cuantitativas, llevadas a cabo en los años 2002, 2006 y 2009. En esta investigación, se utilizarán los datos la cohorte menor de la ronda 3 , es decir, niños entre 8 y 9 años. Cabe anotar que se cuenta con información acerca de los padres, de la vivienda, del rendimiento de los niños y de los puntajes de las pruebas administradas en la ronda 2, cuando los niños tenían entre 5 y 6 años de edad. Adicionalmente, para contar con información acerca de la escuela, se usa la base de datos del Censo Escolar de 2009, que es realizado anualmente por el Ministerio de Educación. El censo cuenta con información reportada por los directores de cada escuela acerca de la matrícula, docentes, infraestructura de la escuela, ambientes educativos, acceso a servicios básicos, entre otros. Esta base de datos se recogió por primera vez en 1993; desde 1998, se realiza anualmente.

\subsection{Tamaño de la muestra efectiva}

La muestra original fue recolectada en veinte comunidades distintas en todo el país, que fueron elegidas de manera aleatoria, de la misma manera que las viviendas dentro de cada comunidad. Dado que es un estudio sobre pobreza infantil, se excluyó de la muestra al 5\% de los distritos más ricos (Escobal y Flores, 2008). La tabla I presenta el tamaño de la muestra efectiva, es decir, la utilizada en el análisis. En ella, se observa que el 17\% de los alumnos asiste a 112 escuelas privadas; y el 83\%, a 244 escuelas públicas. 
Tabla I. Tamaño de la muestra efectiva ${ }^{6}$

\begin{tabular}{lrrrr}
\hline \multicolumn{1}{c}{ Tipo de escuela } & \multicolumn{2}{c}{ Alumnos } & \multicolumn{2}{c}{ Escuelas } \\
\hline I.E. pública & 763 & $83 \%$ & 244 & $69 \%$ \\
I.E. privada & 156 & $17 \%$ & 112 & $31 \%$ \\
\hline Total & 919 & $100 \%$ & 357 & $100 \%$ \\
\hline
\end{tabular}

Fuente: Niños del Milenio (2009)

Elaboración propia

\subsection{Variables}

Los datos que analizaremos provienen de los siguientes instrumentos administrados en las rondas 2 y 3 de Niños del Milenio y del Censo Escolar.

\section{Variables dependientes}

- Test de Vocabulario en Imágenes Peabody (PPVT): Es una prueba de desarrollo cognitivo que mide el vocabulario receptivo de personas de entre tres y diecinueve años. El examinador muestra 4 imágenes a la persona pidiéndole que señale la imagen que mejor representa la palabra dicha; consta de 125 ítems que se aplican de acuerdo con la edad del niño. Siguiendo la teoría clásica, se usará el puntaje bruto, que puede tomar valores entre 0 a 125, para poder comparar el puntaje con la ronda anterior y utilizar un modelo de valor agregado.

- Prueba de matemática: Se usa el puntaje bruto que consta de veintinueve ítems; es decir, toma valores de cero a veintinueve, que mide nociones básicas cuantitativas y numéricas. Del total de preguntas, nueve ítems son sobre conocimientos de los números y operaciones básicas; y veinte ítems, sobre sumas, restas, multiplicaciones y divisiones. Ambas pruebas fueron aplicadas en 2009, cuando los niños tenían entre 7 y 8 años de edad.

\section{Variable independiente de interés}

Refiere a la variable dicotómica, que indica el tipo de gestión educativa de la escuela, tomando el valor uno si es privada; o cero, si es pública.

6. El tamaño de la muestra se redujo, debido a que, en la muestra total del estudio (1943 niños en el año 2009), no se incluyeron a los niños que vivían en zonas rurales (550) y algunos niños no tenían información acerca de su escuela (360). Con aquellos restantes, que no contaban con información completa, se realizó un test de medias comparando esa muestra con la muestra efectiva, no se encontraron diferencias significativas entre ambas. 


\section{Características de la familia y el niño}

- Lengua materna castellano: Es una variable cualitativa, que toma el valor de uno si la lengua materna del niño es castellano; o cero, si es indígena. Se espera que tenga una relación positiva con el rendimiento, porque gran parte de los materiales, cuadernos, libros de texto están en español, lo cual dificulta el entendimiento de los niños cuya lengua materna es indígena.

- Años de educación de la madre: Según la literatura, afecta positivamente a los niños, puesto que madres más educadas pueden brindarles un mejor acompañamiento y apoyo a sus hijos en las tareas.

- Edad en meses: Tendría una relación positiva, pues, a medida que el niño va creciendo, va aprendiendo más cosas.

- Sexo: Es una variable cualitativa, que toma el valor de uno si es niño; y cero, si es niña.

- Número de hermanos: Se espera que afecte de manera negativa el rendimiento, debido a que, mientras más hermanos tenga el niño, menor será la inversión en educación de los padres en su hijo.

- Horas de estudio fuera de la escuela: Esta variable influye en el rendimiento, en la medida que la práctica de ejercicios mejora el entendimiento de las clases y, por ende, su rendimiento.

- Edad a la que empezó la escuela: Esta variable puede afectar su rendimiento de manera negativa, dado que reflejaría un atraso en el niño al ingresar a la escuela.

- Índice de bienestar? Es un índice formado por tres subíndices: calidad de la vivienda medida, según los materiales de la casa; acceso a servicios básicos; y bienes de consumos duraderos, estimado mediante el número de bienes durables en el hogar. Se espera que dicho índice esté asociado positivamente con el rendimiento del niño.

- Puntajes previos en PPVT/CDA (2006): Es el puntaje rezagado del PPVT administrado cuando el niño tenía alrededor de cinco años (para el modelo del PPVT) o el puntaje en el CDA, prueba que consta de quince preguntas que miden nociones cuantitativas. En este examen, se le muestra al niño una serie de imágenes y se le pide que elija aquella que mejor representa la descripción que brinda el examinador (para el modelo de Matemática).

\section{Características de la escuela}

Estas variables se obtuvieron del padrón y Censo Escolar (Unidad de Estadística Educativa - Escale, 2009).

7. Dada la alta correlación entre el índice de bienestar y el tipo de gestión educativa, se corrió un modelo bivariado (índice de bienestar $=\alpha 0+\alpha 1$ Tipo de gestión $+\varepsilon i$ ), y nos quedamos con los residuos para tener el índice de bienestar libre del efecto o correlación con el tipo de gestión escolar. Esta técnica se puede utilizar cuando se cuenta con variables altamente correlacionadas, que pueden sesgar el modelo (Behrman y Duc, 2014). 
- Proporción de profesores hombres en la I.E.: Puesto que la manera de enseñar de las profesoras está más orientada al trabajo activo del niño en el aula, al trabajo grupal y a un mejor clima en el aula, se espera una relación negativa de esta variable con respecto al rendimiento del niño (Escardíbul y Mora, 2013; Chudgar y Sankar, 2008).

- I.E. es polidocente completa: Esta variable toma el valor de uno si en la escuela hay un aula para cada grado; o cero, si es una I.E. multigrado. Esto último refiere a que, en una misma aula, se enseña a varios grados, por lo que se puede retrasar el aprendizaje de los alumnos.

- Acceso a servicios básico: Corresponde a un índice de cero a tres, que indica si tiene acceso a electricidad, agua y desagüe.

- Índice de infraestructura: Toma valores de cero a tres, estimado mediante el material de los pisos (valor de uno si es de cemento), techos (uno si es de concreto armado) y paredes (uno si es de cemento).

- Mobiliario escolar: Es un índice que toma valores de cero a dos, que intenta medir los mínimos materiales necesarios para un correcto dictado de clases. En ese esquema, toma el valor de cero si es que carece de pizarras y de suficientes carpetas para el alumnado; uno, si no cuenta con una de esas características; y dos, si es que cuenta con los dos recursos mínimos necesario para la enseñanza. Estos últimos tres índices dan cuenta de las condiciones escolares en las que los niños estudian, las cuales afectan sus oportunidades de aprendizaje y, por ende, su rendimiento, como señalan algunos estudios (Fisher, 2000; Cuyvers et ál., 2011).

\subsection{Estrategia empírica ${ }^{8}$}

Según el modelo propuesto por Todd y Wolpin (2003), el logro de los niños depende de insumos de la familia y de la escuela. Así, $T_{i j a}$ representa el logro del niño i que vive en el hogar $\mathrm{j}$ a la edad $\mathrm{a}, \mathrm{S}_{\mathrm{ij}}$ (a) es el vector de insumos de la familia hasta la edad, $\mu_{\mathrm{ij}}$ es el vector de insumos de la escuela, $\varepsilon_{\mathrm{ija}}$ es la habilidad innata y es el error de medición. De esta manera, la función es la siguiente:

$$
\mathrm{T}_{\mathrm{ija}}=\mathrm{T}_{\mathrm{a}}\left[\mathrm{F}_{\mathrm{ij}}(\mathrm{a}), \mathrm{S}_{\mathrm{ij}}(\mathrm{a}), \mu_{\mathrm{ij} 0}, \varepsilon_{\mathrm{ija}}\right]
$$

Modelar de manera adecuada el rendimiento o aprendizaje de los individuos es uno de los principales retos en el campo de la educación, dado que existe una gran cantidad de variables difíciles de observar y medir, como la habilidad innata de los niños, su motivación, la ayuda que les dan sus padres en sus tareas, entre otras. Glewwe (2002) menciona que, si bien algunas de estas variables pueden medirse, contar con una base de datos que incluya toda esta información es poco probable. Esta omisión de variables genera que existan sesgos en la estimación, es decir, endogeneidad. Así, el autor plantea que una de

8. Todos los análisis fueron hechos utilizando el software Stata 12. 
las soluciones utilizadas en la literatura es usar los modelos de valor agregado, que son aquellos que tienen como dependiente el puntaje de una prueba e incluyen, como independiente, el puntaje de la prueba que fue tomada años atrás, es decir, el puntaje rezagado.

En este estudio, incluimos el puntaje del PPVT y de matemática, que fueron evaluaciones tomadas cuando el niño tenía entre siete y ocho años. El puntaje anterior o rezagado es de la prueba administrada cuando el niño tenía entre cuatro y cinco años; es decir, el modelo corrige y agrega el rendimiento en la infancia temprana hasta que inicia la escuela, que es aproximadamente a los seis años. Para obtener una estimación más precisa del efecto, se tomaron en cuenta a aquellos niños que no cambiaron de colegio entre la ronda 2 (2006) y la ronda 3 (2009). Como menciona Glewwe (2002), si la primera prueba fue aplicada de manera correcta, esta captura el efecto de las inversiones de los padres y del hogar. En ese sentido, el modelo de valor agregado soluciona parcialmente el problema de endogeneidad, puesto que no habría variables omitidas.

Con la finalidad de responder al primer objetivo, sobre si existen diferencias en el rendimiento producto de asistir a escuelas públicas o a privadas, usaremos un modelo de valor agregado, usando cluster a nivel de institución educativa para evitar los posibles problemas de correlaciones al haber niños agrupados en una misma escuela. Incluimos el puntaje del PPVT rezagado en el modelo y el puntaje del CDA para el caso de matemática.

$$
\text { PPVT }_{\mathrm{ia}}=\beta_{0}+\beta_{1} \text { IEprivada }+\mathrm{X}_{\mathrm{ia}}^{\prime} \alpha+\mathrm{Z}_{\mathrm{ia}}^{\prime} \theta+\gamma \mathrm{PPVT}_{\mathrm{i}, \mathrm{a}-1}+\eta_{\mathrm{ia}} \ldots \text { (a) }
$$

Donde:

\begin{tabular}{|c|c|c|}
\hline $\mathrm{PPVT}_{\mathrm{ia}}$ & $=$ & Puntaje en la prueba PPVT del niño i, a la edad a \\
\hline $\mathrm{PPVT}_{\mathrm{i}, \mathrm{a}-1}$ & $=$ & Puntaje en la prueba PPVT del niño i, a la edad (a-1) \\
\hline$\beta_{1}$ & $=$ & $\begin{array}{l}\text { Coeficiente entre el puntaje del PPVT y el tipo de gestión de } \\
\text { la escuela }\end{array}$ \\
\hline $\mathrm{X}_{\mathrm{ia}}^{\prime}$ & $=$ & Insumos de la familia \\
\hline $\mathrm{Z}_{\text {ia }}^{\prime \prime}$ & $=$ & Insumos de la escuela \\
\hline$\alpha$ & $=$ & $\begin{array}{l}\text { Vector de coeficientes entre el puntaje del PPVT y los } \\
\text { insumos de la familia }\end{array}$ \\
\hline$\theta$ & $=$ & $\begin{array}{l}\text { Vector de coeficientes entre el puntaje del PPVT y los } \\
\text { insumos de la escuela }\end{array}$ \\
\hline$\eta_{\text {ia }}$ & $=$ & Error \\
\hline
\end{tabular}

En el segundo modelo a estimar, controlaremos por una prueba administrada en la ronda 2 el CDA (Child Development Assessment ${ }^{9}$ ), que mide nociones cuantitativas básicas de los niños. El modelo a estimar será el siguiente:

9. Si bien las pruebas de matemática y el CDA no son iguales, el CDA es un indicador de habilidades básicas de matemáticas, que mide nociones de cantidad que tiene el niño; de esta manera, se constituye como una aproximación a las habilidades de matemática. 


$$
\text { Matemática }{ }_{\mathrm{ia}}=\beta_{0}+\beta_{1} \mathrm{IEprivada}+\mathrm{X}_{\mathrm{ia}}^{\prime} \alpha+\mathrm{Z}_{\mathrm{ia}}^{\prime} \theta+\gamma \mathrm{CDA}_{\mathrm{i}, \mathrm{a}-1}+\eta_{\mathrm{ia}} \ldots . \text { (b) }
$$

Donde:

\begin{tabular}{|c|c|c|}
\hline $\mathrm{PPVT}_{\text {ia }}$ & $=$ & Puntaje en la prueba PPVT del niño i, a la edad a \\
\hline $\mathrm{CDA}_{\mathrm{i}, \mathrm{a}-1}$ & $=$ & Puntaje en la prueba del CDA del niño i a la edad (a-1) \\
\hline$\beta_{1}$ & $=$ & $\begin{array}{l}\text { Coeficiente entre el puntaje de matemática y el tipo de } \\
\text { gestión de la escuela }\end{array}$ \\
\hline $\mathrm{X}_{\mathrm{ia}}^{\prime}$ & $=$ & Insumos de la familia \\
\hline $\mathrm{Z}_{\text {iล }}^{\prime}$ & $=$ & Insumos de la escuela \\
\hline$\alpha$ & $=$ & $\begin{array}{l}\text { Vector de coeficientes entre el puntaje de matemática y los } \\
\text { insumos de la familia }\end{array}$ \\
\hline$\theta$ & $=$ & $\begin{array}{l}\text { Vector de coeficientes entre el puntaje de matemática y los } \\
\text { insumos de la escuela }\end{array}$ \\
\hline$\eta_{\text {ia }}$ & $=$ & Error \\
\hline
\end{tabular}

\section{Resultados}

\subsection{Descriptivos}

El puntaje en la prueba de Peabody (PPVT) es, en promedio, menor para los niños que asisten a escuelas públicas: como muestra la figura I, en el año 2006, el promedio en ese caso era de 31,1 puntos, mientras que los niños que asisten a escuelas privadas tenían un promedio de 45,1 . Asimismo, 3 años después, la diferencia era de 9 puntos: 71,6 y 62,1 para los niños que asisten a públicas y privadas, respectivamente. Si bien la brecha se redujo cinco puntos, la brecha aún existe.

Figura I. Puntajes brutos en el PPVT en las dos rondas, según el tipo de gestión de la escuela

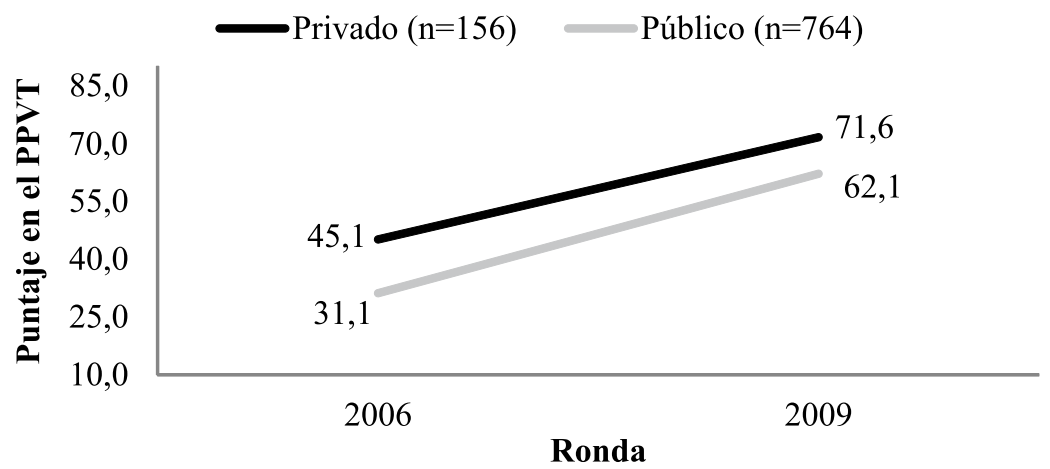

Las diferencias entre público y privado son significativas para el año 2006 y 2009.

Fuente: Niños del Milenio (2009)

Elaboración propia 
La tabla II presenta las características principales de los alumnos de la muestra, según el tipo de gestión educativa. Los niños tienen prácticamente la misma edad y la misma proporción de hombres. El 96\% de los alumnos de escuelas públicas tiene como lengua materna el castellano, mientras que todos los que asisten a escuelas privadas tienen como primera lengua el español. En promedio, los años de escolaridad de las madres de escuelas públicas es 8,4 (secundaria incompleta), mientras que los años de escolaridad en el privado es 11,8 (secundaria completa). Los niños con menos hermanos y más horas de dedicación al estudio asisten a escuelas privadas. La diferencia en el índice de bienestar es de 0,17 a favor de las instituciones educativas privadas.

Tabla II. Características individuales según el tipo de gestión de la escuela

\begin{tabular}{lccc}
\hline Variables & $\begin{array}{c}\text { Público } \\
(\mathrm{n}=763)\end{array}$ & $\begin{array}{c}\text { Privado } \\
(\mathrm{n}=156)\end{array}$ & $\begin{array}{c}\text { Total } \\
(\mathrm{n}=919)\end{array}$ \\
\hline Edad en meses & 94,91 & 94,21 & 94,79 \\
Hombre & 0,49 & 0,50 & 0,50 \\
Lengua materna castellano & 0,96 & 1,00 & 0,97 \\
Años de educación de la madre & 8,41 & 11,78 & 8,99 \\
Número de hermanos & 1,73 & 1,11 & 1,62 \\
Índice de bienestar & 0,58 & 0,75 & 0,61 \\
Horas de estudio fuera de la escuela & 2,07 & 2,48 & 2,14 \\
Edad a la que empezó la escuela & 6,35 & 6,27 & 6,33 \\
\hline
\end{tabular}

Fuente: Niños del Milenio (2009)

Elaboración propia

Con respecto a las características de la escuela, la tabla III muestra que la proporción de hombres en las escuelas públicas es de $31 \%$, mientras que, en las escuelas privadas, es de 19\%. Los índices de acceso a servicios básicos e infraestructura son más altos para las escuelas privadas, lo cual se traduce en mejor material de construcción, acceso a luz, agua y electricidad. Dado que el análisis se realiza en un contexto urbano, para ambas escuelas, el porcentaje de colegios polidocentes completos -es decir, un grado y un profesor por aula- es mayor al 90\%. Finalmente, sobre el mobiliario escolar, en promedio, los colegios privados están mejor equipados que los públicos. 
Tabla III. Características de la escuela según el tipo de gestión de la escuela

\begin{tabular}{lccc}
\hline Variables & $\begin{array}{c}\text { Público } \\
(\mathrm{n}=244)\end{array}$ & $\begin{array}{c}\text { Privado } \\
(\mathrm{n}=112)\end{array}$ & $\begin{array}{c}\text { Total } \\
(\mathrm{n}=357)\end{array}$ \\
\hline Proporción de profesores hombres & 0,31 & 0,19 & 0,27 \\
Acceso a servicios básicos & 2,70 & 2,94 & 2,78 \\
Índice de infraestructura & 2,18 & 2,46 & 2,26 \\
I.E. polidocente completa & 0,92 & 0,91 & 0,92 \\
Mobiliario escolar & 1,45 & 1,97 & 1,62 \\
\hline
\end{tabular}

Acceso a servicios básicos: Indica si la escuela tiene acceso a luz, agua y desagüe, tomando valores de 0 a 3 . Índice de infraestructura: Indica si el material de la pared, techo y piso es de cemento, tomando valores de 0 a 3. Mobiliario escolar: Indica si la escuela tiene al menos los materiales mínimos como pizarra y carpetas, tomando valores de 0 a 2.

Fuente: Niños del Milenio (2009)

Elaboración propia

\subsection{Asociación entre rendimiento y tipo de gestión escolar}

Para responder al primer objetivo de la investigación, la tabla IV presenta los resultados de los modelos multivariados, que estiman el efecto que tiene asistir a una escuela privada sobre el rendimiento de los niños. Los coeficientes han sido estandarizados, es decir, presentados en desviaciones estándar con respecto a la variable dependiente. Los modelos siguen el orden presentado en las tablas IV y V con la finalidad de resaltar la ventaja de usar una base longitudinal que permite estimar modelos de valor agregado, en lugar de realizar un análisis de corte transversal, puesto que, si no se controlara por el puntaje previo, los resultados podrían conllevar a conclusiones incorrectas.

En cuanto al PPVT, el primer modelo plantea la asociación entre el tipo de escuela y el puntaje en vocabulario de los niños; el coeficiente muestra que el asistir a una escuela privada aumenta el PPVT en 0,28 desviaciones estándar. Dado que este resultado puede deberse a otras características, incluimos otras variables que moderarán el efecto del tipo de gestión educativa. El modelo 2 incluye las características individuales y de la familia ${ }^{10}$ : el asistir a una escuela privada aún es positivo y significativo; sin embargo, la magnitud del efecto disminuye. El modelo 3 añade las características de la escuela ${ }^{11}$, que modera el efecto del tipo de escuela, pero sigue siendo significativo.

10. Entre estas características, se encuentran edad en meses, sexo, lengua materna, años de educación de la madre, número de hermanos, índice de bienestar, horas de estudio fuera de la escuela y edad a la que empezó la escuela.

11. Estas características corresponden a proporción de profesores hombres en la escuela, acceso a servicios básicos, índice de infraestructura, polidocente completa y mobiliario escolar. 
El último modelo es el de valor agregado e incluye el puntaje previo en el PPVT, que controla por todas las inversiones hechas hasta esa edad. Las variables que tienen un efecto significativo en el modelo son el género $-10 \%$ a favor de los hombres- y los años de educación de la madre $-10 \%$, puesto que un año más de educación puede incrementar el puntaje del PPVT en 0,27 desviaciones-. El índice de bienestar es significativo al $10 \%$ y, como muestra la literatura, su relación con el rendimiento es positiva (ver anexo I). El número de hermanos tiene una relación negativa con el rendimiento del niño. La proporción de profesores hombres en la escuela tiene una relación negativa, lo cual está alineado con estudios previos que encuentran resultados similares. Finalmente, los resultados muestran que el tipo de gestión no tiene un efecto significativo sobre el vocabulario receptivo del niño, lo cual puede deberse al tipo de prueba que se está analizando. La prueba del Peabody mide el vocabulario y su administración solo requiere que el niño señale una figura; en ese sentido, no evalúa competencias relacionadas a la lectura, reflexión, entre otras. De la misma manera, dado que es un modelo de valor agregado, el incremento del vocabulario se puede dar en contextos que no estén asociados necesariamente a la escuela, puesto que en el día a día el lenguaje de los niños puede mejorar a partir de las prácticas en el hogar, las actividades que realiza el niño fuera de la escuela, entre otras.

Con respecto a matemática, la primera columna de la tabla $\mathrm{V}$ presenta el modelo bivariado, en el cual el efecto del tipo de gestión educativa sobre el rendimiento es de 0,26 desviaciones estándar. El modelo 2 incluye las características de la familia; si bien estas reducen la magnitud del efecto del tipo de escuela, este último sigue siente positivo y significativo. El tercer modelo añade las características de la escuela. Finalmente, en el último modelo, las características significativas son la edad, el sexo (a favor de los hombres), la educación de la madre, el índice de bienestar, las horas de estudio fuera de la escuela, la edad a la que empezó la escuela -aspecto que tiene un signo negativo, puesto que, mientras mayor sea la edad, mayor será el atraso escolar- y la proporción de profesores hombres en la escuela -que, también, es significativo y negativo(ver anexo II).

La variable de interés en el modelo, a diferencia del PPVT, permanece significativa, aun luego de controlar por el puntaje previo en matemática con un coeficiente de 0,12 desviaciones estándar, el cual es considerado como un efecto leve según la literatura (Sapelli y Vial, 2002). Esto puede deberse a que el rendimiento de matemática está más orientado por la currícula escolar; es decir, depende en gran medida de características de la escuela. Como señalan algunos estudios, el nivel de profundidad con el que se trabaja el conocimiento matemático en la escuela está altamente asociado a los buenos resultados de los estudiantes (Cueto, Ramírez, León y Pain, 2003; Cueto, Ramírez, León y Guerrero, 2004). A diferencia del rendimiento en la prueba de vocabulario, el rendimiento en matemática se encuentra más ligado a la escuela y a los procesos pedagógicos dentro de ella. 
Tabla IV. Efecto de asistir a una escuela privada sobre el rendimiento de los niños (Coeficientes estandarizados)

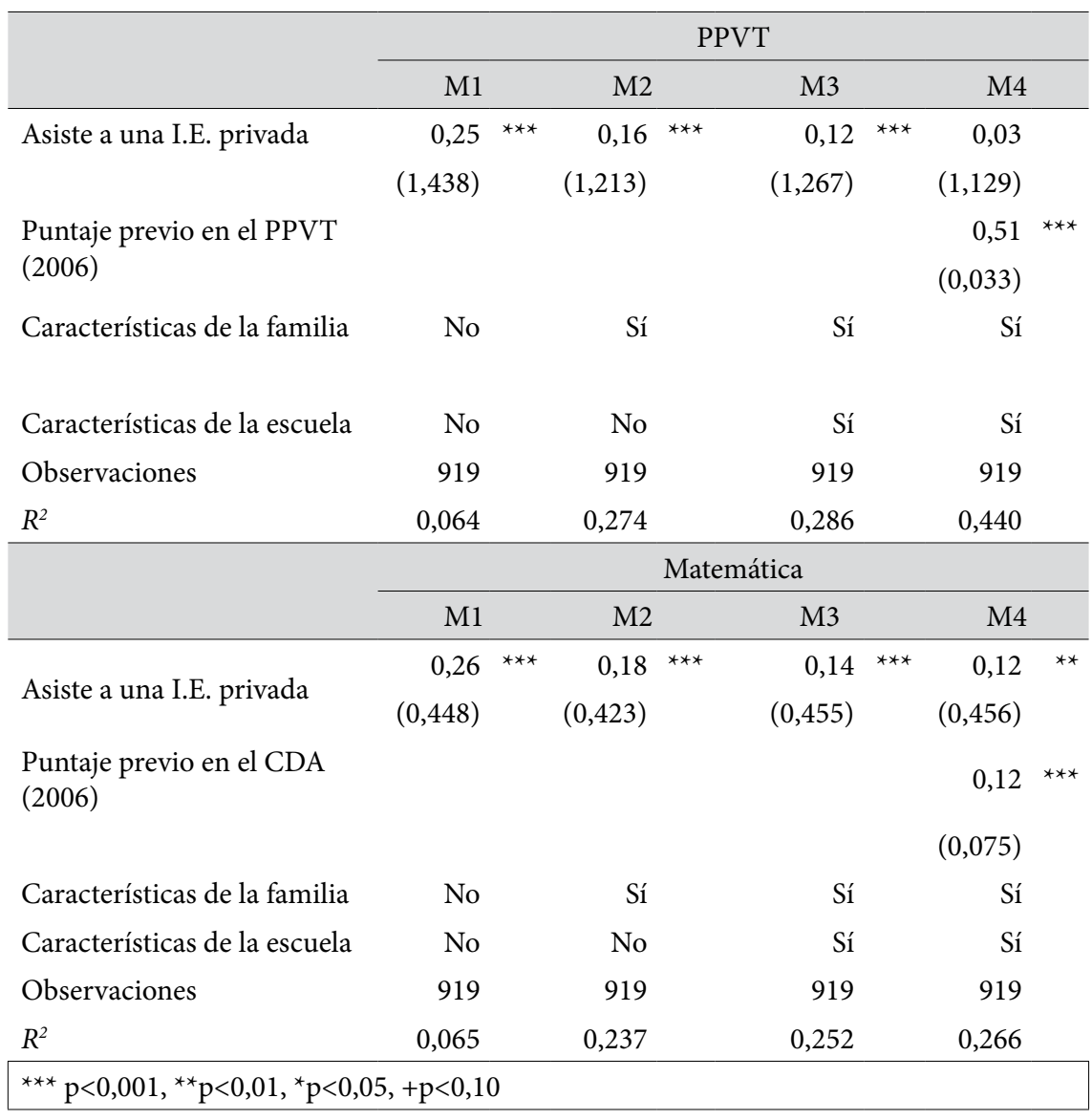

Nota: Los coeficientes están estandarizados para observar la importancia relativa de cada variable con respecto a la dependiente. Los errores estándar entre paréntesis están ajustados por posibles correlaciones entre alumnos que asisten a la misma escuela.

Fuente: Elaboración propia

\section{3 ¿Qué factores explican estas diferencias en el rendimiento?}

Para responder el segundo objetivo, descomponemos la brecha de rendimiento para entender qué factores -de la escuela o de la familia- explican en mayor proporción estas diferencias. La tabla $\mathrm{V}$ muestra qué porcentaje de la varianza es explicada por el conjunto de características. En los primeros modelos, se observa que las variables de la familia explican la tercera parte de la diferencia en el rendimiento de asistir a una escuela privada o pública y las características 
de la escuela explican en un 16\%. Sin embargo, al incluir el valor del rezago, los porcentajes cambian de manera drástica, puesto que este valor controla por todas las inversiones previas en la primera infancia, lo cual explica el 63\% de las diferencias. Las variables asociadas al niño y la familia explican el 16\%; y las asociadas a la escuela, el $8 \%$.

Tabla V. Descomposición de la brecha del PPVT

\begin{tabular}{|c|c|c|c|c|c|c|c|c|}
\hline \multirow[b]{3}{*}{ Descomposición de brecha } & \multicolumn{8}{|c|}{ Descomposición de la brecha } \\
\hline & \multicolumn{2}{|c|}{ Modelo 1} & \multicolumn{2}{|c|}{ Modelo 2} & \multicolumn{2}{|c|}{ Modelo 3} & \multicolumn{2}{|c|}{ Modelo 4} \\
\hline & $\beta$ & $\%$ & $\beta$ & $\%$ & $\beta$ & $\%$ & $\beta$ & $\%$ \\
\hline Sin explicar & 9,58 & $100,0 \%$ & 5,99 & $62,5 \%$ & 4,71 & $49,1 \%$ & 1,21 & $12,6 \%$ \\
\hline $\begin{array}{l}\text { Características del niño y } \\
\text { de la familia }\end{array}$ & - & - & 3,59 & $37,5 \%$ & 3,38 & $35,3 \%$ & 1,55 & $16,2 \%$ \\
\hline Características de la escuela & - & - & - & - & 1,49 & $15,5 \%$ & 0,80 & $8,3 \%$ \\
\hline Puntaje previo PPVT & - & - & - & - & - & - & 6,06 & $63,2 \%$ \\
\hline
\end{tabular}

Fuente: Niños del Milenio (2009)

Elaboración propia

La tabla VI presenta el mismo análisis, pero, para el caso de matemática. Como se observa, los resultados confirman lo hallado previamente; es decir, las variables de la escuela están más asociadas al rendimiento en matemática que al vocabulario del niño, lo cual explica el $18 \%$ de las diferencias. Como se mencionó, el desempeño en matemática depende del colegio y de los procesos pedagógicos que se siguen en esta; no obstante, por limitaciones de la base, no se cuenta con información acerca de estos procesos, por lo que casi la mitad de la brecha no puede explicarse en el modelo.

Tabla VI. Descomposición de la brecha de matemática

\begin{tabular}{|c|c|c|c|c|c|c|c|c|}
\hline \multirow[b]{3}{*}{ Descomposición de brecha } & \multicolumn{8}{|c|}{ Descomposición de la brecha } \\
\hline & \multicolumn{2}{|c|}{ Modelo 1} & \multicolumn{2}{|c|}{ Modelo 2} & \multicolumn{2}{|c|}{ Modelo 3} & \multicolumn{2}{|c|}{ Modelo 4} \\
\hline & $\beta$ & $\%$ & $\beta$ & $\%$ & $\beta$ & $\%$ & $\beta$ & $\%$ \\
\hline Sin explicar & 3,05 & $100,0 \%$ & 2,17 & $71,2 \%$ & 1,64 & $53,8 \%$ & 1,47 & $48,2 \%$ \\
\hline $\begin{array}{l}\text { Características del niño y } \\
\text { de la familia }\end{array}$ & - & - & 0,88 & $28,9 \%$ & 0,80 & $26,2 \%$ & 0,74 & $24,3 \%$ \\
\hline Características de la escuela & - & - & - & - & 0,61 & $20,0 \%$ & 0,55 & $18,0 \%$ \\
\hline Puntaje previo CDA & - & - & - & - & - & - & 0,29 & $9,5 \%$ \\
\hline
\end{tabular}

Fuente: Niños del Milenio (2009)

Elaboración propia 


\section{Conclusiones y recomendaciones}

Uno de los principales objetivos del país, en cuanto a educación se refiere, es garantizar el acceso a la educación y que esta sea de buena calidad. Se podría decir que el primero de estos objetivos se está alcanzado; sin embargo, si bien la calidad educativa -en términos de rendimiento de los niños- ha aumentado en los últimos años, aún queda un espacio por mejorar. Esta investigación se basa en el análisis de uno de los factores asociados al rendimiento, el tipo de gestión de la escuela, pública o privada.

Los resultados muestran que, en el modelo bivariado, la relación entre el rendimiento y el tipo de gestión es significativa y a favor de los alumnos de escuelas privadas. No obstante, al incluir como control el puntaje previo del niño, el asistir a una escuela privada ya no afecta el vocabulario receptivo (PPVT), aunque sí influye en el rendimiento en matemática $(0,12 \mathrm{SD}, \mathrm{p}<0,05)$. Esto puede ser consecuencia de la naturaleza de la prueba que se aplica: el rendimiento en la prueba de vocabulario no depende exclusivamente de la escuela, sino que abarca contenidos que pueden aprenderse en el día a día de los niños mediante la interacción con la familia o sus pares. Para el caso de matemática, el mejorar su rendimiento depende en mayor medida del currículo implementado en las escuelas. Debido a esto, se encontró que las variables asociadas a la institución educativa explican en mayor proporción las diferencias en matemática (18\%) que las diferencias en el PPVT (8\%). En ese marco, una de las fortalezas de este estudio es que no solo llena un vacío en la literatura acerca de la asociación del tipo de gestión escolar y el rendimiento, sino que -al hacer uso de una base longitudinal- se puede controlar por puntajes previos del niño, a diferencia de los estudios de corte transversal, que no cuentan con información acerca del pasado.

Cabe resaltar que, dado que el estudio Niños del Milenio es pro pobre, se excluyó de su marco muestral al 5\% de los distritos más ricos de país; debido a ello, la muestra de escuelas privadas se encuentra subestimada, y la brecha entre los que asisten a una escuela pública o privada podría ser mayor. Asimismo, si bien se cuenta con información acerca de la escuela, una limitación del modelo es que no se incluyó ninguna variable vinculada a procesos educativos. Por ejemplo, en el caso de matemática, el $48 \%$ de la brecha no se pudo explicar, lo cual podría deberse a la omisión de estas variables que son claves para el aprendizaje en matemática. Cueto, León, Sorto y Miranda (s.f. $b$ ), y Guadalupe, León y Cueto (2013) muestran que, aunque la educación de los docentes es relevante en el aprendizaje de los estudiantes, el conocimiento pedagógico de los profesores es un factor clave en la enseñanza de matemática en las aulas. Con respecto a la metodología, si bien el problema de endogeneidad solo ha sido corregido de manera parcial mediante el uso de un modelo de valor agregado, otros métodos de estimación que controlan el sesgo de selección han mostrado que el efecto de asistir a una escuela privada continúa siendo positivo y significativo (Valdivia, 2003). 
De acuerdo con los resultados encontrados, son los factores asociados al individuo y sus familias los que explican en mayor medida las diferencias en el rendimiento; no obstante, aun cuando los factores escolares explican en menor proporción estas diferencias, igual, se genera un espacio en el cual la política educativa puede actuar. En términos de recomendación de política, es necesario cerrar las brechas de insumos entre las escuelas. Los resultados muestran que las privadas cuentan con mejores servicios y ambientes que las públicas, lo cual refuerza las inequidades, puesto que son los estudiantes con menos recursos los que suelen asistir a estas escuelas y, finalmente, quienes obtienen promedios más bajos, a partir de lo cual se forma un círculo de inequidades (Cueto, León y Miranda, 2015). Por otro lado, si bien los niveles de rendimiento han progresado de manera positiva, los resultados de las escuelas privadas han disminuido: las cifras nacionales del año 2014 muestran que, en matemática, prácticamente no hay diferencias entre ambos tipos de gestión escolar. Ante esta situación, se hace necesario mayor control y regulación de la educación privada por parte del Estado. También, se encontró que gran parte de la brecha en matemática no se puede explicar, lo cual es posible que responda a la limitación de las variables asociadas a procesos, es decir, lo que realmente hacen los docentes en el aula. Frente a este escenario, es importante continuar con el soporte pedagógico brindado a los docentes, quienes juegan un rol relevante en la enseñanza.

En términos de investigación en educación, se sugiere continuar esta línea de investigación, en la que se puedan incluir variables de procesos educativos que permitan ver el efecto de la labor pedagógica que se realiza en el aula de clase y, por ende, en las escuelas. Asimismo, es relevante elaborar estudios que diferencien el efecto de asistir a una escuela privada según la calidad de esta, puesto que se sabe que, hoy en día, la heterogeneidad de las escuelas privadas es cada vez mayor. Finalmente, es relevante mejorar las escuelas públicas del país con miras a brindar las mismas oportunidades educativas a todos los estudiantes, de forma tal que se pueda cerrar las brechas de rendimiento, $y$ brindarles las mismas oportunidades a futuro a todos los niños y niñas del país.

\section{Nota biográfica}

Alejandra Miranda es magíster en Economía por la Pontifica Universidad Católica del Perú y bachiller en Economía por la misma casa de estudios. Se desempeña como investigadora asistente del área de Educación y Aprendizajes en Grade. Sus principales áreas de interés son educación y equidad, desarrollo económico y evaluación de impacto. 


\section{Referencias}

Behrman, J. y Duc, L. T. (2014). Early-childhood growth faltering, postinfancy recovery and educational outcomes in late childhood: Evidence from Vietnam (GCC Working Paper Series, GCC 14-04).

Chávez, J. C. (2001). Determinación de los factores explicativos de los resultados escolares de la educación primaria en el Perú (tesis para optar el grado de Magíster en Gestión y Políticas Públicas). Universidad de Chile, Santiago de Chile.

Chudgar, A. y Sankar, V. (2008). The relationship between teacher gender and student achievement: Evidence from five Indian states. Compare, 38(5), 627-642.

Coleman, J. S., Hoffer, T. B. y Kilgore, S. (1982). High school achievement: Public, catholic, and other private schools compared. New York: Basic Books.

Cuenca, R. (2013). La escuela pública en Lima Metropolitana. ¿Una institución en extinción? Revista Peruana de Investigación Educativa, 5, 73-98

Cueto, S. (2011). Hábitos, estrategias y rendimiento en comprensión de lectura en PISA 2009 (Documento de trabajo). Grade, Lima.

Cueto, S., León, J. y Miranda, A. (2015) Peru: Impact of socioeconomic gaps in educational outcomes. En S. Schwartzman, Education in South America (385-404). Londres: Bloomsbury.

Cueto, S., León, J., Miranda, A. y Vásquez, M. (s.f. a). Education trajectories: From early childhood to early adulthood in Peru (documento no publicado). Young Lives, Oxford University, Oxford.

Cueto, S., León, J., Sorto, A. y Miranda, A. (s.f. b). Teachers pedagogical content knowledge and mathematics achievement of students in Peru (documento no publicado). Grade, Lima.

Cueto, S., Ramírez, C., León, J. J. y Pain, O. (2003). Oportunidades de aprendizaje $y$ rendimiento en matemática en una muestra de estudiantes de sexto grado de primaria de Lima (Documento de trabajo No 43). Grade, Lima.

Cueto, S., Ramírez, C., León, J. y Guerrero, G. (2004). Oportunidades de aprendizaje y rendimiento en matemática de los estudiantes de tercer y cuarto grados de primaria en Lima y Ayacucho. En M. Benavides (Ed.), Educación, procesos pedagógicos y equidad (15-68). Lima: Grade.

Cuyvers, K., De Weerd, G., Dupont, S., Mols, S. y Nuytten, C. (2011). Wellbeing at school: Does infrastructure matter? (Working/Policy Paper). CELE Exchange 2011/10. OECD, París.

Escardíbul, J. O. y Mora, T. (2013). Teacher gender and student performance in mathematics. Evidence from Catalonia (Spain). Journal of Education and Training Studies, 1(1), 39-46. 
Escobal, J. y Flores, E. (2008). An assessment of the Young Lives sampling approach in Peru (Young Lives Technical Note No. 3). University of Oxford, Oxford.

Fisher, K. (2000). Building better outcomes. The impact of school infrastructure on student outcomes and behavior. Schools issues digest. Department of Education, Training and Youth Affairs, Australian Government, Sydney.

Glewwe, P. (2002). Schools and skills in developing countries: Education Policies and socioeconomic outcomes. Journal of Economic Literature, 40(2), 436-482.

Guadalupe, C., León, J. y Cueto, S. (2013). Charting progress in learning outcomes in Peru using National Assessments (Background paper prepared for the Education for All Global Monitoring Report 2013/4). Unesco, Lima.

Guerrero, G., León, J., Rosales, E., Zapata, M., Freire, S., Saldarriaga, V. y Cueto, S. (2012). Young Lives school survey in Peru: Design and initial findings. Young Lives (Working Paper $\mathrm{N}^{\circ}$ 92). University of Oxford, Oxford.

Jiménez, E., Lockheed M. E. y Paqueo, V. (1991). The relative efficiency of private and public schools in developing countries. The World Bank Research Observer, 6(2), 205-218.

Learning Metrics Task Force LMTF (2013). Toward universal learning: Recommendations from the Learning Metrics Task Force. Montreal y Washington, D. C.: Unesco, Institute for Statistics and Center for Universal Education at the Brookings Institution.

León, J., Díaz, J., Benavides, M. (2015). Una mirada a la educación privada en el Perú y sus efectos en el rendimiento de los estudiantes peruanos (versión preliminar). Lima: Banco Interamericano de Desarrollo.

McEwan, P. (2001). The effectiveness of public, catholic and non religious private school in Chile's voucher system. Education Economics, 9(2), 103128.

Miranda, A. (2015). La asociación entre el tipo de gestión escolar y el aprendizaje de los estudiantes en el Perú: Magnitud y moderadores de su efecto (Tesis de maestría sin publicar). Lima: Pontificia Universidad Católica del Perú.

Mizala, A., Romaguera, P. y Reinaga, T. (1999). Factores que inciden en el rendimiento escolar en Bolivia (Documento de trabajo $n^{\circ}$ 61). Centro de Economía Aplicada, Universidad de Chile, Santiago de Chile.

Mizala, A. y Romaguera, P. (2000). School performance and choice. The Chilean experience. Santiago de Chile: Centro de Economía Aplicada, Universidad de Chile.

Montero, C. y Cuenca, R. (2008). Sobre notas y aprendizajes escolares: Opiniones $y$ demandas de la población en el Perú. Lima: Foro Educativo. 
OECD (2013). PISA 2012 results in focus: What 15-year-olds know and what they can do with what they know. París: OECD Publishing. doi:10.1787/9789264208780-en

Psacharopolus, G. (1986). Public vs. private schools in developing countries: Evidence from Colombia and Tanzania. International Journal of Educational Developmen, 7(1), 59-67.

Rydeen, J. (2009). Test case: Do new schools mean improved test scores? American School \& University, 81(13). New York: Penton Media Inc. y American School \& University.

Sapelli, C. y Vial, B. (2002). The performance of private and public schools in the Chilean voucher system. Cuadernos de Economía, Latin American Journal of Economics, 39(118), 423-454.

Schneider, M. (2002). Do school facilities affect academic outcomes? Washington, D.C.: National Clearinghouse for Educational Facilities. Recuperado de http://www.edfacilities.org/pubs/outcomes.pdf

Todd, P., y Wolpin, K. (2003). On the specification and estimation of the production function for cognitive achievement. The Economic Journal, 113, 3-33.

Tooley, J. y Dixon, P. (2003). Private schools for the poor: A case study from India. Reading, UK: Centre for British Teachers.

Tooley, J., Dixon, P., y Gomathi, S. V. (2007). Private schools and the millennium development goal of universal primary education: A census and comparative survey in Hyderabad, India. Oxford Review of Education, 539-560.

Unesco (2014). Primera entrega de resultados Terce - Tercer Estudio Regional Comparativo y Explicativo. Santiago de Chile: Orealc/Unesco Santiago.

Unidad de Estadística Educativa - Escale (s.f.). Escale. Estadística de la Calidad Educativa. Recuperado de www.escale.minedu.gob.pe

Oficina de Medición de la Calidad de los Aprendizajes y Grupo de Análisis para el desarrollo (2000). Resultados de las pruebas de Matemáticas y Lenguaje. ¿Qué aprendimos a partir de la evaluación Crecer 1998? Boletín 5/6. Lima: Ministerio de Educación.

Oficina de Medición de la Calidad de los Aprendizajes (2004). Factores asociados al rendimiento estudiantil. Resultados de la Evaluación Nacional 2001 (Documento de trabajo ${ }^{\circ}$ 9). Oficina de Medición de la Calidad de los Aprendizajes del Ministerio de Educación, Lima.

(2005). Evaluación Nacional del Rendimiento Estudiantil 2004. Informe descriptivo de resultados (Documento de Trabajo 12). Ministerio de Educación, Lima.

(2015). Resultados Generales 2007-2014. Oficina de Medición de la Calidad Educativa - Ministerio de Educación. Recuperado de http://umc.minedu.gob.pe 
Valdivia, P. (2003). El efecto de la escuela privada sobre el rendimiento estudiantil (tesis de Maestría). Instituto de Economía de la Pontificia Universidad Católica de Chile, Santiago de Chile. Recuperado de http://www.economia. puc.cl/docs/tesis_pvaldivia.pdf

Wu, K. B. (2009). Peru: Education at a crossroads: Challenges and opportunities for the 21st century. Washington D.C.: Banco Mundial. 


\section{Anexos: Resultados de los modelos estimados}

Anexo I. Modelo multivariado del efecto del tipo de gestión educativa en el PPVT

\begin{tabular}{|c|c|c|c|c|c|c|c|c|}
\hline \multirow{3}{*}{ Puntaje previo en PPPVT (2006) } & \multicolumn{8}{|c|}{ PPVT } \\
\hline & \multicolumn{2}{|c|}{ M1 } & \multicolumn{2}{|l|}{ M2 } & \multicolumn{2}{|l|}{ M3 } & \multicolumn{2}{|c|}{ M4 } \\
\hline & & & & & & & 0,431 & $* * *$ \\
\hline & & & & & & & $(0,033)$ & \\
\hline \multirow[t]{2}{*}{ Asiste a una I. E. privada } & 9,584 & $* * *$ & 5,990 & $* * *$ & 4,706 & $* * *$ & 1,213 & \\
\hline & $(1,438)$ & & $(1,213)$ & & $(1,267)$ & & $(1,129)$ & \\
\hline \multirow[t]{2}{*}{ Edad en meses } & & & 0,363 & $\star * *$ & 0,353 & $* * *$ & 0,0591 & \\
\hline & & & $(0,083)$ & & $(0,085)$ & & $(0,085)$ & \\
\hline \multirow[t]{2}{*}{ Sexo (hombre) } & & & 1,888 & * & 2,046 & * & 1,251 & + \\
\hline & & & $(0,821)$ & & $(0,812)$ & & $(0,707)$ & \\
\hline \multirow[t]{2}{*}{ Lengua materna castellano } & & & 1,914 & & 0,849 & & 1,461 & \\
\hline & & & $(2,298)$ & & $(2,354)$ & & $(2,187)$ & \\
\hline \multirow[t]{2}{*}{ Años de educación de la madre } & & & 0,766 & $* * *$ & 0,735 & $* * *$ & 0,277 & * \\
\hline & & & $(0,118)$ & & $(0,114)$ & & $(0,112)$ & \\
\hline \multirow[t]{2}{*}{ Número de hermanos } & & & $-0,980$ & ** & $-0,911$ & ** & -0556 & + \\
\hline & & & $(0,344)$ & & $(0,344)$ & & $(0,300)$ & \\
\hline \multirow[t]{2}{*}{ Índice de bienestar } & & & 19,82 & $* * *$ & 17,37 & $* * *$ & 5,340 & + \\
\hline & & & $(3408)$ & & $(3,462)$ & & $(2,748)$ & \\
\hline \multirow[t]{2}{*}{ Horas de estudio fuera de la escuela } & & & 1,173 & ** & 1,097 & ** & 0,473 & \\
\hline & & & $(0,421)$ & & $(0,420)$ & & $(0,389)$ & \\
\hline \multirow[t]{2}{*}{ Edad a la que empezó la escuela } & & & $-1,335$ & & $-1,383$ & + & $-0,811$ & \\
\hline & & & $(0,831)$ & & $(0,811)$ & & $(0,764)$ & \\
\hline \multirow{2}{*}{$\begin{array}{l}\text { Proporción de prof. hombres en la } \\
\text { I.E. }\end{array}$} & & & & & $-8,013$ & ** & $-4,601$ & + \\
\hline & & & & & $(2,847)$ & & $(2,562)$ & \\
\hline \multirow[t]{2}{*}{ Acceso a servicios básicos } & & & & & 0,503 & & 0,651 & \\
\hline & & & & & $(1,246)$ & & $(1,122)$ & \\
\hline \multirow[t]{2}{*}{ Índice de infraestructura } & & & & & $-0,339$ & & $-0,909$ & \\
\hline & & & & & $(0,942)$ & & $(0,856)$ & \\
\hline \multirow[t]{2}{*}{ I.E. polidocente completa } & & & & & 1,463 & & 0,231 & \\
\hline & & & & & $(2,187)$ & & $(2,007)$ & \\
\hline \multirow[t]{2}{*}{ Mobiliaria Escolar } & & & & & 0,724 & & 0,436 & \\
\hline & & & & & $(0,784)$ & & $(0,742)$ & \\
\hline \multirow[t]{2}{*}{ Constante } & 62,05 & $* * *$ & 26,13 & * & 28,11 & * & 44,60 & $* * *$ \\
\hline & $(0,983)$ & & $(10,65)$ & & $(11,48)$ & & $(10,70)$ & \\
\hline \multirow{2}{*}{$\begin{array}{l}\text { Observaciones } \\
R^{2}\end{array}$} & 919 & & 919 & & 919 & & 919 & \\
\hline & 0,064 & & 0,274 & & 0,286 & & 0,440 & \\
\hline
\end{tabular}

Errores estándar entre paréntesis

${ }^{\star * *} \mathrm{p}<0,001 ;{ }^{* *} \mathrm{p}<0,01 ;{ }^{\star} \mathrm{p}<0,05,+\mathrm{p}<0,1$ 
Anexo II. Modelo multivariado del efecto del tipo de gestión educativa en Matemática

\begin{tabular}{|c|c|c|c|c|c|c|c|c|}
\hline & & & $\mathrm{Ma}$ & temát & & & & \\
\hline & M1 & & M2 & & M3 & & M4 & \\
\hline Puntaje previo en CDA (2006) & & & & & & & $\begin{array}{r}0,285 \\
(0,075)\end{array}$ & $* * *$ \\
\hline Asiste a una I. E. privada & $\begin{array}{r}3,049 \\
(0,448)\end{array}$ & $* * *$ & $\begin{array}{r}2,164 \\
(0,423)\end{array}$ & $* * *$ & $\begin{array}{r}1,638 \\
(0,455)\end{array}$ & $* * *$ & $\begin{array}{r}1,468 \\
(0,456)\end{array}$ & ** \\
\hline Edad en meses & & & $\begin{array}{r}0,185 \\
(0,036)\end{array}$ & $* * *$ & $\begin{array}{r}0,185 \\
(0,035)\end{array}$ & $* * *$ & $\begin{array}{r}0,164 \\
(0,037)\end{array}$ & $* * *$ \\
\hline Sexo (hombre) & & & $\begin{array}{r}0,551 \\
(0,293)\end{array}$ & + & $\begin{array}{r}0,609 \\
(0,291)\end{array}$ & * & $\begin{array}{r}0,590 \\
(0,291)\end{array}$ & * \\
\hline Lengua materna castellano & & & $\begin{array}{r}-0,006 \\
(0,968)\end{array}$ & & $\begin{array}{l}-0,487 \\
(0,972)\end{array}$ & & $\begin{array}{r}-0,506 \\
(0,957)\end{array}$ & \\
\hline Años de educación de la madre & & & $\begin{array}{r}0,219 \\
(0,045)\end{array}$ & $* * *$ & $\begin{array}{r}0,208 \\
(0,044)\end{array}$ & $* * *$ & $\begin{array}{r}0,193 \\
(0,044)\end{array}$ & $* * *$ \\
\hline Número de hermanos & & & $\begin{array}{r}-0,0804 \\
(0,115)\end{array}$ & & $\begin{array}{r}-0,0576 \\
(0,113)\end{array}$ & & $\begin{array}{r}-0,0341 \\
(0,111)\end{array}$ & \\
\hline Índice de bienestar & & & $\begin{array}{r}4,304 \\
(0,940)\end{array}$ & $* * *$ & $\begin{array}{r}3,328 \\
(0,959)\end{array}$ & $* * *$ & $\begin{array}{r}2,746 \\
(0,984)\end{array}$ & ** \\
\hline $\begin{array}{l}\text { Horas de estudio fuera de la } \\
\text { escuela }\end{array}$ & & & $\begin{array}{r}0,282 \\
(0,133)\end{array}$ & * & $\begin{array}{r}0,252 \\
(0,129)\end{array}$ & + & $\begin{array}{r}0,238 \\
(0,127)\end{array}$ & + \\
\hline Edad a la que empezó la escuela & & & $\begin{array}{r}-1,353 \\
(0,265)\end{array}$ & $* * *$ & $\begin{array}{r}-1,356 \\
(0,268)\end{array}$ & $* * *$ & $\begin{array}{r}-1,326 \\
(0,264)\end{array}$ & $* * *$ \\
\hline & & & & & $-2,391$ & * & $-2,223$ & * \\
\hline $\begin{array}{l}\text { Proporción de prof. hombres en } \\
\text { la I.E. }\end{array}$ & & & & & $(1,069)$ & & $(1,068)$ & \\
\hline Acceso a servicios básicos & & & & & $\begin{array}{r}0,273 \\
(0,301)\end{array}$ & & $\begin{array}{r}0,281 \\
(0,302)\end{array}$ & \\
\hline Índice de Infraestructura & & & & & $\begin{array}{r}0,007 \\
(0,291)\end{array}$ & & $\begin{array}{r}-0,029 \\
(0,291)\end{array}$ & \\
\hline I.E. polidocente completa & & & & & $\begin{array}{r}0,461 \\
(0,569)\end{array}$ & & $\begin{array}{r}0,484 \\
(0,559)\end{array}$ & \\
\hline Mobiliaria Escolar & & & & & $\begin{array}{r}0,377 \\
(0,231)\end{array}$ & & $\begin{array}{r}0,327 \\
(0,228)\end{array}$ & \\
\hline Constante & $\begin{array}{r}12,350 \\
(0,276)\end{array}$ & $* * *$ & $\begin{array}{r}0,802 \\
(3,875)\end{array}$ & & $\begin{array}{r}0,477 \\
(4,034)\end{array}$ & & $\begin{array}{r}0,050 \\
(3,970)\end{array}$ & \\
\hline $\begin{array}{l}\text { Observaciones } \\
R^{2}\end{array}$ & $\begin{array}{r}919 \\
0,065\end{array}$ & & $\begin{array}{r}919 \\
0,237\end{array}$ & & $\begin{array}{r}919 \\
0,252\end{array}$ & & $\begin{array}{r}919 \\
0,266\end{array}$ & \\
\hline
\end{tabular}

Errores estándar entre paréntesis 